\title{
PET/CT in paediatrics: it is time to increase its use!
}

\author{
Isabel Roca • Marc Simó • Constantino Sábado • \\ Josep Sanchez de Toledo
}

Published online: 18 January 2007

(C) Springer-Verlag 2007

In adults, ${ }^{18} \mathrm{~F}$-FDG PET and PET/CT have proved to be useful tools in oncological patients [1]. For example, it has been reported that PET can modify the initial staging in about $40 \%$ of patients with lymphoma, leading to modification of therapy in $18 \%$ of them [2]. Similar results in terms of clinical management, i.e. changes in staging and treatment, have been described in large series of patients with other malignancies. PET and PET/CT have also been shown to exert a high impact on oncological management in the paediatric population [35]. However, the low incidence of the paediatric malignancies has made it difficult to elucidate the precise role of these examinations and to attain evidence-based indications for them. Nevertheless, more and more papers have been published demonstrating that FDG PET/CT is also useful in children. Results obtained in paediatric lymphoma patients have shown high rates (up to 23\%) of therapy changes owing to FDG PET results [6], and several recent papers have described the usefulness of this technique in most other paediatric malignancies, such as neuroblastoma and sarcomas.

This editorial commentary refers to the article http://dx.doi.org/ 10.1007/s00259-006-0253-9.

I. Roca $(\bowtie) \cdot$ M. Simó

Servei de Medicina Nuclear, Hospital Universitari Vall d'Hebron, Barcelona, Spain

e-mail: rocabielsa@yahoo.es

C. Sábado $\cdot$ J. S. de Toledo

Servei d'Oncologia Pediàtrica,

Hospital Universitari Vall d'Hebron,

Barcelona, Spain
What is the situation regarding correlative image? In both children and adults it is clear that imaging diagnosis is more accurate when anatomical and metabolic images are interpreted together. Hybrid equipment and fusion imaging (and PET/CT is probably the reference) provide better information than is acquired by separate anatomical and metabolic examinations or even by comparison of them. This fact is encouraging imaging specialists (in CT, MRI, nuclear medicine, etc.) to collaborate more closely, thereby increasing communication, improving reports and increasing the clinical impact of imaging modalities $[1,2]$.

Several papers, even in paediatric oncology, have reported that fused PET/CT improves diagnostic accuracy compared with PET alone or side by side comparison of PET and CT images [6, 7]. An excellent example of this benefit is given in a recently published paper from the German Society of Paediatric Oncology and Hematology on Hodgkin's disease. This prospective, multicentre study compares the accuracy of conventional imaging methods and FDG PET in the initial staging of paediatric patients diagnosed with Hodgkin's lymphoma. Twenty-one nodal and six extranodal regions were evaluated by conventional imaging methods and FDG PET independently, and later the images were also compared side by side and fused. Results were statistically superior when anatomical regions were evaluated using both methods in combination. No differences were observed according to the way in which the methods were combined, i.e. side by side viewing or image fusion. However, the confidence of analysts in the results was clearly superior when the fusion method was used [8].

In this issue, Zvi Bar-Sever et al. describe the results of a prospective study on 46 consecutive paediatric patients who underwent ${ }^{18} \mathrm{~F}$-FDG PET/CT for known or suspected 
malignancies. Blinded interpretation of PET and PET/CT is described, comparing the results between the modalities (results being classified as malignant, equivocal or benign) and then with the biopsy findings or the clinical outcome. Discordant results of PET and PET/CT were obtained in $22 \%$ of the studies: PET/CT was negative in all the negative PET studies and was classified as definitely positive or negative in most of the equivocal PET studies. Interestingly, PET/CT ruled out malignancy in $20 \%$ of the equivocal or positive PET studies. PET/CT showed a sensitivity of $92 \%$ (similar to other series) and a specificity of $78 \%$ for diagnosis of childhood malignancy. This lower specificity is probably related to the high number $(n=20)$ of false positive PET/CT studies in nine patients with lymphoma (in whom there was a high incidence of viral infections and thymic rebound). This study confirms the added value of PET/CT compared with PET alone, as well as the usefulness of PET/CT in paediatric patients. The authors have contributed in expanding our knowledge of the use of PET/CT in paediatric patients.

Although in some areas of Nuclear Medicine it is feared that the mingling of functional imaging procedures and anatomical imaging procedures from the world of Radiology could threaten Nuclear Medicine, the specialty is in fact being reinforced by the rapid expansion of PET/CT. The use of newer tracers, in both oncological and non- oncological illnesses, foretells an interesting future: we should be on our guard.

\section{References}

1. Schiepers C, Filmont JE, Czernin J. PET for staging of Hodgkin's disease and non-Hodgkin's lymphoma. Eur J Nucl Med Mol Imaging 2003;30(Suppl 1):S82-8.

2. Naumann R, Beuthien-Baumann B, Reiss A, Schulze J, Hanel A, Bredow J, et al. Substantial impact of FDG PET imaging on the therapy decision in patients with early-stage Hodgkin's lymphoma. Br J Cancer 2004;90(3):620-5.

3. Shulkin BL. PET applications in pediatrics. Q J Nucl Med 1997 Dec;41(4):281-91.

4. Hahn K, Pfluger T. Has PET become an important clinical tool in paediatric imaging? Eur J Nucl Med Mol Imaging 2004;31 (5):615-21.

5. Roca I, Simo M, Sanchez de Toledo J. Clinical impact of PET in pediatrics. Rev Esp Med Nucl 2004;23(5):359-68.

6. Wegner EA, Barrington SF, Kingston JE, Robinson RO, Ferner RE, Taj $\mathrm{M}$, et al. The impact of PET scanning on management of paediatric oncology patients. Eur J Nucl Med Mol Imaging 2005;32 (1):23-30.

7. Jager PL, Slart RH, Corstens F, Oyen WJ, Hoekstra O, Teule J. PET-CT: a matter of opinion? Eur J Nucl Med Mol Imaging 2003;30(3):470-1.

8. Furth C, Denecke T, Steffen I, Ruf J, Voelker T, Misch D, et al. Correlative imaging strategies implementing CT, MRI, and PET for staging of childhood Hodgkin disease. J Pediatr Hematol Oncol 2006;28(8):501-2. 